\title{
The Role and Influence of Artificial Intelligence on Advertising Industry
}

\author{
Yongqi $\mathrm{Yu}$
}

University of International Business and Economics, Beijing, 100105, China

yyq2000321@163.com

\begin{abstract}
Since the industrial revolution, significant advancements in technological innovation have resulted in the transformation of numerous manual tasks and processes that had existed for decades, to the point where artificial intelligence (AI) technologies are widely used in advertising to increase efficiency and meet market demand. Artificial intelligence (AI) refers to computers becoming more capable of fulfilling particular jobs and activities presently done by people in the workplace and across society. The effect of artificial intelligence on the advertising process is becoming a growing concern, manifesting as a systematic restructuring. In comparison to conventional advertisement creation, AI offers several significant benefits, as shown in this research. This study provides important and relevant insight into artificial intelligence technology and its implications for the future of business and society while also acknowledging the social and industrial influences on the speed and direction of AI development. As artificial intelligence permeated every link in the advertising industry, It plays a positive role in the development of industry, is able to replace inefficient manual labor and improves the overall efficiency of industry. The paper points out that the market still needs to be aware of the disadvantages of artificial intelligence, considering some of the moral risks and privacy concerns. Relevant departments should further investigate these issues.
\end{abstract}

Keywords: Artificial intelligence, Big data, Advertising, Sentiment analysis

\section{INTRODUCTION}

Perhaps the most exciting of all the technological developments in advertising is artificial intelligence (AI). It has gained significant attention in the advertising sector globally, and this focus is projected to rise significantly as technology advances. Numerous issues remain hidden beneath the excitement: What is artificial intelligence advertising exactly, and how does AI affect the advertising process?

AI has the potential to fundamentally disrupt the advertising industry, affecting every aspect of the business[1]. Professionals worldwide place a premium on AI and machine learning in advertising and marketing[2]. The industry discourse reveals an overwhelming favorable attitude regarding the use of artificial intelligence in advertising.

According to Shelly[3], several figures demonstrate widespread use: Over $75 \%$ of users have used an AI-enabled service or device (Adobe 2018). By 2021, AI marketing is expected to grow at a 53 percent compound annual growth rate. 2020 (Gingerich). By 2023 (Enberg 2019), global digital advertising is expected to reach $\$ 517.51$ billion, with AI accounting for $80 \%$ of it (Ad Exchanger 2019).

The remaining sections of this paper are organized as follows: Section two presents numerous definitions of AI from the expert contributors and overall themes within the literature; Section three details the multiple applications of $\mathrm{AI}$ in the advertising industry and many of the key debates; Section four presents a discussion on analysis of how AI is transforming the advertising business by several perspectives; Section five presents ethical issues of AI-driven advertising industry. Lastly, the study is concluded in Section six.

\section{ACADEMIC DEFINITIONS OF AI}

Numerous definitions of AI have been proposed in the literature, each encapsulating the central concepts of non-human intelligence programmed to perform specific tasks. When artificial intelligence first emerged, it was defined as a breed of robots that was "rapidly evolving, a breed capable of seeing, reading, speaking, learning, 
and even feeling emotions"[4]. Later on, as it grew in popularity, academic definitions became more plentiful. Ransbotham et al. (2017) define AI as the theory and development of computer systems capable of performing tasks generally requiring human intelligence, such as visual perception, speech recognition, decision-making, and language translation (Oxford Dictionary, p. 2). Kaplan and Haenlein[5] provide a more detailed and perhaps elaborate description of AI in terms of its ability to independently interpret and learn from external data to achieve specific outcomes via flexible adaptation.

The common denominator among these definitions is that machines are increasingly capable of performing specific roles and tasks currently performed by humans in the workplace and in society at large(2019)[8]. Individuals can teach machines to behave similarly to humans. We can endow them with the faculties of sight, hearing, speech, movement, and writing. These machines would be called artificial intelligence (AI) if they could then teach themselves to improve their vision, hearing, speaking, movement, and writing-all without human intervention. That is precisely where we stand today in terms of AI capabilities. Individuals encountered an AI tool or AI model that sees, hears, speaks, moves, and writes using speech recognition, computer vision, natural language processing, AI chatbots, or another AI capability. These tools then improve at those tasks on their own, without being explicitly programmed to do so.

\section{AI APPLICATION IN THE ADVERTISING INDUSTRY}

The impact of AI on the advertising process is an increasing challenge that takes the form of a systematic reorganization. A new set of process steps-consumer insight discovery, ad creation, media planning, and buying, and ad impact evaluation-has emerged[6], resulting in the emergence of a new class of advertising activities, including large-scale personalized advertising production based on consumer profiling, omnichannel precision media planning, and buying, and proactive strategy-based algorithms that evaluate and optimize advertising impact.

\subsection{AD Platform}

Programmatic advertising exchanges and ad tech platforms manage real-time buying and selling using artificial intelligence and machine learning. This includes almost every ad exchange, third-party network, and advertising solution available on Facebook, Instagram, and Snapchat.

These exchanges, services, and platforms are unlikely to explain to marketers how their artificial intelligence works in the near future. That, however, is precisely the point: Even behind the scenes, artificial intelligence dictates how money is spent, who sees commercials, and the overall efficacy of campaigns. That implies that if a business uses paid to advertise, it must be conversant with the vocabulary of artificial intelligence (this resource is a fantastic place to start) and ask the right questions about how each ad platform's AI may affect the spending.

The following is a clear illustration of this: Facebook advertising, more precisely, the frequency and relevancy score of advertisements. These two metrics are essential pieces of data that Facebook's algorithms use to calculate how much money to spend on advertising and how the commercials are shown totally without human participation.

Increased exposure may not necessarily translate into superior outcomes. According to conventional advertising research, the optimal advertising frequency is at least three exposures during the course of a brand's purchasing cycle. According to conventional advertising wisdom, people should "strike" the target as frequently as possible with the same campaign. On the other hand, repeated exposure on Facebook may work against the effort.This is because the algorithms that determine how Facebook adverts are shown take user input into account. If individuals view the advertisement too frequently and give inadequate evaluations, the relevancy score may decline. According to Social Media Examiner, "in most cases, a higher frequency correlates with a lower relevancy score."A high relevance score suggests that the advertisement is more likely to be displayed to the desired audience than competing advertisements. As a result, performance is improved, and costs are reduced. AI algorithms that run today's ad exchanges utilize intricate interplays of data points to affect advertisers' campaign performance and budget effectiveness.

\subsection{Optimize Budget and Targeting}

Today, artificial intelligence solutions show that they can optimize advertising expenditure and target automatically. AI can analyze the ad spend and targeting data, then determine which actions (spending adjustments, targeting modifications) will improve performance. Across a large number of complex initiatives, these actionable insights are produced and executed at scale. We discovered that RedBalloon, a travel company, used an artificial intelligence (AI) application called Albert to optimize and manage their digital advertising budget and plan automatically. The outcomes were mind-blowing. Albert discovered methods for increasing return on investment and outperforming human agencies by optimizing expenditure and targeting. Additionally, through the insights gained from adequate amounts of expenditure data, the tool discovered new consumers for 
RedBalloon's products that the firm was unaware existed.

Human-powered and conventional software-based advertising systems were beaten by AI's ability to learn and grow without human involvement, resulting in a major competitive advantage for the business.

\subsection{Ad Creation and Management}

AI determines how the advertisements work and may even assist people in significantly increasing performance. However, it is also utilized nowadays to simplify the process of creating advertising campaigns significantly.

Platforms with advertising components, most notably Facebook, offer AI that enables people to generate ad text and variants far quicker than people could manually, utilizing previously submitted material.

However, some commercially accessible technologies go much further. The tool with AI capabilities Advertisements on Facebook and Instagram are created by Phrasee and are optimized for conversion based on what has worked in the past for the business. As it learns from each new ad, the technology becomes better and better over time.(This is the same kind of AI technology that drives use cases for content generation in content marketing.)

Additionally, an AI system may help people save time managing their entire marketing strategy. WordStream combines machine learning with the flexibility to make adjustments to many ad campaigns on Facebook, Google, and Bing with a few clicks[7].

\section{ANALYSIS OF HOW AI IS TRANSFORMING THE ADVERTISING BUSINESS}

Compared to traditional advertising production, artificial intelligence technology has enhanced the effectiveness of advertising production and marketing, making brand marketing more human, accurate, effective, and improving the effectiveness of advertising communication and information contact rate.

\subsection{More advertising content creation and higher marketing efficiency with lower production costs}

Production of advertising using artificial intelligence technology can categorize, combine sources of information, rapidly create new ideas, and execute intelligent marketing. Therefore, it increases advertising creative generating efficiency while also drawing more users' attention and increasing advertising effectiveness and conversion rate while also lowering advertising industry production costs.
Using artificial intelligence technology, marketers can better target their ads by selecting and combining the most effective time, place, scene, and medium and analyzing multiple target users to identify platform-specific factors such as scenario use habits and consumption preferences. This allows them to keep costs under control while simultaneously improving the marketing impact.

\subsection{Greater advertising impact and information reach rate}

Brand marketing will become more precise and successful as artificial intelligence (AI) technology advances in the advertising business. Advertisement content relevant to the target audience may be created from vast and fragmented resources using artificial intelligence and content marketing, for example.

As of 2018, the OPPO phone is based on the latest data-driven technology to provide users with information such as personal consumption data analysis and classification, user behavior and shopping consumption, and APP use. This is all done through algorithm programs, data flows, mobile terminal market software, and relevant searches. The target market is identified once the user picture has been created early in the brand marketing process. Further stages of data services are provided to empower target audiences and increase the pertinence of advertising.

\subsection{Better access to information and consuming experience for users}

Artificial intelligence has significantly enhanced the user experience as an extension of the human body, intellect, cognition, and emotion. For instance, in 2016, Baidu partnered with Mercedes Benz to host an "augmented reality show." Users only search for "Mercedes-Benz E-class" in the Baidu App to have an immersive visual experience of the vehicle gently "driving out" from the mobile terminal device.

A high level of user engagement defines interactive advertising: As an audience-focused type of advertising, interactive impact advertising is often presented in the form of games or competitions to add interest and entice people to engage; High advertising effectiveness and conversion rate: when compared to traditional or native advertising, interactive advertising's content innovation is more personalized and targeted, and is, therefore, more likely to encourage users to interact with the advertising, thereby increasing the click conversion rate; Strong monetization capability: Interactive advertising does not compete with traditional types of advertising, but instead encourages higher conversion rates based on current user traffic and offers additional revenue possibilities. 


\section{ETHICAL ISSUES OF AI-DRIVEN ADVERTISING INDUSTRY}

The primary concern is the privacy and security of the user's personal information. Commercial interests predominate in the use of AI technology, which is still in its early stages. Many network platforms do not adequately secure user data, posing severe threats to data security and the growth of the advertising business.

When gathering user identification information, artificial intelligence will amass much personal information about them. Information gathered from various sources, such as user-registered data on websites or platforms, cameras, and GPS positioning systems used by popular image recognition apps is challenging to interpret. Users' personal information is included in these data mashups. Many pieces of information are taken, utilized, and resold without the knowledge or permission of the people who originally collected it.

The first is the protection of user privacy and the security of data. Artificial intelligence technology is still in its infancy, and business considerations mainly drive it. Many network platforms do not adequately secure user data, posing significant hidden risks to data security and the growth of the advertising sector alike.

Using artificial intelligence to gather a user's identification information will result in collecting a vast quantity of personal data. Users' information recorded on websites or platforms, cameras and GPS location of critical applications of visual recognition systems, etc. are all examples of data sources. These data assemblages include personal information about the users. Many users' data is taken, misused, and marketed without their knowledge or permission.

Secondly, there is an issue in the intelligent advertising industry where ad giants unfairly compete against one another. Advertising head platform has a significant market share now that the Matthew effect has emerged. However, this brings up a new issue: the unequal distribution of internet advertising's growth. By distinguishing in vertical sectors and depending on artificial intelligence and 5G technology to enhance competitiveness in the short video industry, BAT, an Internet behemoth, attempts to create a matrix of short video platforms. Increasing their ad market share while also grabbing attention resources is the goal of any advertising behemoth. The first is the protection of user privacy and the security of data. Artificial intelligence technology is still in its infancy, and business considerations mainly drive it. Many network platforms do not adequately secure user data, posing significant hidden risks to data security and the growth of the advertising sector alike.

Lastly, there is the problem of intelligent advertising's copyright. However, even though algorithm developers build and create works on algorithm processes, artificial intelligence robots are no longer simply an extension of human-made technological instruments. Big data technology and algorithm procedures aided by deep learning are constantly evolving and deriving this complicated development.

Artificial intelligence robots create the original material produced by extensive data learning, not the system's engineers. Nevertheless, there is a disagreement here as well. The academic community is still researching and speeding the development of relevant legislation by the norm of originality of works.

\section{CONCLUSION}

The existing definitions are sorted out and summarized, and the essential components of AI definitions in academic circles are extracted. Secondly, the paper illustrates how AI is applied in the advertising industry by taking examples of facts, which can be divided into four aspects, including large-scale personalized advertising production based on consumer profiling, omnichannel precision media planning, and buying, and proactive strategy-based algorithms that evaluate and optimize advertising impact. The paper also discusses how AI changes the current advertising industry, which can be divided into three aspects: enhancing the effectiveness of advertising production and marketing, making brand marketing more human, accurate, effective, and improving the effectiveness of advertising communication and information contact rate.

Although artificial intelligence has permeated every aspect of the advertising industry, the paper emphasizes the importance of the market remaining aware of the disadvantages of artificial intelligence, particularly in light of some of the moral risks and privacy concerns. Relevant departments should continue to improve relevant management policies and work to regulate the application and development of artificial intelligence in the advertising industry. Lastly, other areas in the advertising market should also be examined. Is artificial intelligence affecting jobs in the advertising industry? How may moral risk be avoided? What is the best way to split and establish copyright issues? Is there a distinction between using artificial intelligence for online and offline shopping? Future study should dive further into the answers to these issues and the influence of AI on advertising.

\section{REFERENCES}

[1] Qin X, Jiang Z. The Impact of AI on the Advertising Process: The Chinese Experience[J].

[2] Guttmann, A. Views on AIs influence on advertising performance in the U.S. 
2018.Statista.https://www.statista.com/statistics/87 7560/views-on-ai-improving-advertising-performa nce/

[3] Guttmann, A. Most effective digital marketing techniques 2018. Statista. https://www.statis

[4] Rodgers S . Themed Issue Introduction: Promises and Perils of Artificial Intelligence and Advertising $[\mathrm{J}]$.

[5] Gregory R D . Elastic wave propagation: 1989, editors M. F. McCarthy and M. A. Hayes. Amsterdam: North-Holland Elsevier. pp. $638+$ xviii. Price US\$ 167.75/Dfl. 315.00 ISBN $0-44-87272-8[\mathrm{~J}]$. Journal of Sound \& Vibration, 1990, 143(3):540-540.
[6] Kaplan A, Haenlein M. Rulers of the world, unite! The challenges and opportunities of artificial intelligence[J]. Business Horizons, 2019, 63(1).

[7] Jiang, Zhibin, and Xin Ma (2019), “Applications, Difficulties and Solutions: Advertising Operation under Artificial Intelligence Reconstruction," Journalism and Communication Review, 5, 56-63.

[8] Xia Y, Zhu M, Xin L. The Study of Natural Language Processing Based on Artificial Intelligence[J]. Springer Berlin Heidelberg, 2012.

[9] Dwivedi Y K , Hughes L , Ismagilova E , et al. Artificial Intelligence (AI): Multidisciplinary perspectives on emerging challenges, opportunities, and agenda for research, practice and policy[J]. International Journal of Information Management, 2019, 57(7). 